\title{
Proteinbiosynthese
}

\section{Ordnung am Ribosom - der multifunktionale Komplex NAC}

KARINA GENSE, MARTIN GAMERDINGER

MOLEKULARE MIKROBIOLOGIE, FACHBEREICH BIOLOGIE, UNIVERSITÄT KONSTANZ

\section{Newly synthesized proteins are processed by a variety of ribosome- associated factors that regulate cotranslational protein folding, trans- port and degradation. How these competing factors gain regulated and timely access to ribosomes and specific nascent substrates is poorly understood. Recent studies identified a key factor in eukaryotes that coordinates multiple cotranslational events at the ribosomal tunnel exit - the nascent polypeptide-associated complex (NAC).}

DOI: $10.1007 / \mathrm{s} 12268-020-1367-5$

(C) Die Autoren 2020

In allen Organismen synthetisieren Ribosomen im Cytoplasma der Zelle neue Proteine. Bei diesem als Translation bezeichneten Prozess wird die Nukleotidsequenz eines mRNA-Moleküls in die codierte Aminosäurensequenz eines Polypeptids übersetzt, um ein Protein zu bilden. Die wachsende Polypeptidkette wandert zunächst durch einen engen Tunnel der großen 60S-Ribosomenuntereinheit, bevor sie mit einer Länge von 35-40 Aminosäuren das Cytoplasma erreicht (Abb. 1). Dort angekommen, muss die noch lang gestreckte Polypeptidkette sich in die richtige dreidimensionale Struktur falten und zu ihrem subzellulären Wirkungsort gelangen. Die korrekte Faltung und Lokalisierung neu synthetisierter Proteine wird durch verschiedene Proteinbiogenesefaktoren gesteuert, die dynamisch an den ribosomalen Tunnelausgang binden (Abb. 1, [1]). Die direkte Ribosomenbindung ermöglicht es diesen Faktoren, zum frühestmöglichen Zeitpunkt und noch während der laufenden Proteinsynthese auf ihre Substrate einzuwirken. Sie erkennen spezifisch naszierende Polypeptidsubstrate, unterstützen ihre Faltung direkt im Cytoplasma und lenken ihren ko-translationalen Transport zu Zellorganellen wie dem endoplasmatischen Reticulum (ER) oder den Mitochondrien. Viele der Ribosomen-assoziierten Faktoren konkurrieren um Bindungsstellen am Tunnelausgang, und es ist nicht gut verstanden, wie sie präzise und schnell ihre Substrate auswählen. Jüngste Studien weisen auf einen übergeordneten Faktor in Eukaryoten hin, der ordnend am Tunnelausgang wirkt und andere Proteinbiogenesefaktoren bei der Substratfindung entscheidend unterstützt der nascent polypeptide-associated complex (NAC).

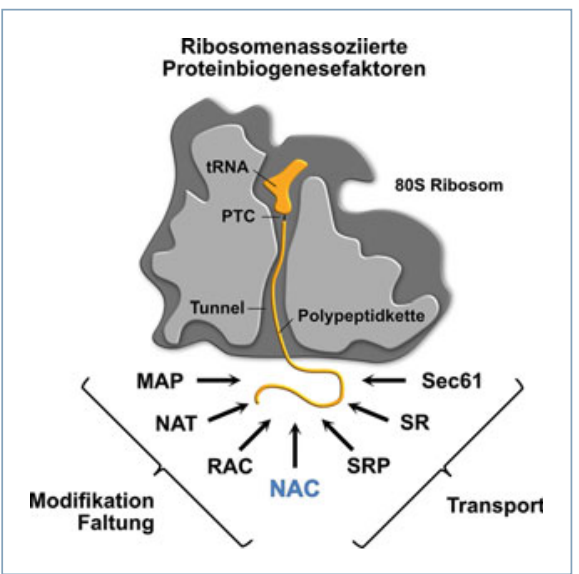

$\Delta$ Abb. 1: Auswahl einiger konkurrierender Proteinbiogenesefaktoren in Eukaryoten, die ko-translational an den ribosomalen Tunnelausgang und Substrate binden. PTC: Peptidyltransferase-Zentrum; tRNA: TransferRNA; MAP: Methionin-Aminopeptidase; NAT: $\mathrm{N}$-Acetyltransferase; RAC: ribosome-associated complex; NAC: nascent polypeptide-associated complex; SRP: signal recognition particle; SR: SRP-Rezeptor; Sec61: ER-Translokon-Komplex.

\section{Nascent polypeptide-associated complex (NAC)}

Einer der Hauptfaktoren in Eukaryoten, der transient mit Ribosomen interagiert, ist der hochkonservierte und ubiquitär exprimierte Proteinkomplex NAC [2]. Der heterodimere Komplex besteht aus einer $\alpha$ - und einer $\beta$-Untereinheit, welche über ihre homologen NAC-Domänen dimerisieren und eine $\beta$-Fassähnliche Struktur bilden (Abb. 2). Die Deletion des NAC-Gens führt zu embryonaler Letalität bei Metazoen, was auf eine grundlegende Haushaltsfunktion in der Proteinbiogenese hinweist. Interessanterweise ist NAC im Vergleich mit anderen Ribosomen-assoziierten Faktoren in sehr hoher Konzentration in der Zelle vorhanden, vergleichbar mit der Konzentration der Ribosomen, was auf ein sehr breites Substratspektrum schließen lässt [1].

Die Ribosomenbindung von NAC ist sehr ungewöhnlich. Der Komplex bindet über die flexiblen N-terminalen Domänen und stellt mehrere alternative Kontakte am Tunnelausgang her (Abb. 2, [3]). Geladene Sequenzmotive in den N-terminalen Domänen interagieren zudem miteinander und regulieren dadurch die Ribosomenbindung von NAC, vermutlich in Abhängigkeit vom jeweiligen Polypeptidsubstrat, das den Tunnelausgang verlässt. Die außergewöhnlichste Eigenschaft von NAC ist jedoch seine Bindung tief im Inneren des ribosomalen Tunnels. Ist dieser nicht von einem Substrat besetzt, fügt NAC die flexible N-terminale Domäne seiner $\beta$-Untereinheit bis in die Nähe des Peptidyltransferase-Zentrums des Ribosoms ein, wo Aminosäuren zu Peptiden verknüpft werden (Abb. 2B, links). Die flexible NAC-Domäne kontaktiert also eine wachsende Polypeptidkette schon bei der Entstehung und begleitet sie zum Tunnelausgang, wo NAC eine alternative Bindung an der Oberfläche der Ribosomen eingeht (Abb. 2B, rechts). Diese Fähigkeit ist einzigartig unter den Ribosomen-assoziierten Proteinbiogenesefaktoren und weist darauf hin, dass NAC sämtliche neu synthetisierten Proteine als erster Faktor bindet. Angesichts all dieser Eigenschaften wird vermutet, dass NAC am Tunnelausgang 
substratspezifische Konformationen annimmt, um Polypeptidsubstrate in verschiedene Biogenese- und Transportpfade zu leiten, wie nachfolgend beschrieben.

\section{Proteintransport}

Der Proteintransport zu Zellorganellen wie dem ER erfolgt ko-translational, indem die translatierenden Ribosomen gezielt zu den Translokationsporen der Organellen gebracht werden. Der Transport zum ER wird über das signal recognition particle (SRP) vermittelt, das spezifisch an hydrophobe Signalsequenzen in Polypeptidketten und an das Ribosom selbst bindet [4]. Nach der Substratbindung interagiert SRP mit dem SRP-Rezeptor (SR), der sich an der ER-Membran befindet und das Ribosom auf den Porenkomplex Sec61 überträgt. SRP und Sec61 binden selektiv hydrophobe Signalsequenzen in ER-Substraten, zeigen aber generell auch eine hohe intrinsische Bindungsaffinität zu Ribosomen, die unabhängig vom synthetisierten Substrat ist. Für eine hohe Transportspezifität ist es daher notwendig, unspezifische Ribosomenkontakte der Transportfaktoren zu unterdrücken, was über den NAC geschieht (Abb. 2B, links) [1, 5]. NAC blockiert wichtige Bindungsstellen für SRP und Sec61 am Tunnelausgang der Ribosomen, die keine ER-Proteine synthetisieren. In Abwesenheit von NAC sind diese Bindungsstellen frei, was $\mathrm{zu}$ unspezifischen SRP- und Sec61-Interaktionen und damit zu fehlerhaftem ER-Proteintransport führt. Wie in einer Studie beim Fadenwurm Caenorhabditis elegans gezeigt wurde, führt der Knock-down von NAC insbesondere zu einer Fehllokalisierung von mitochondrialen Proteinen im ER-Lumen mit fatalen Folgen für die Proteinhomöostase in beiden Organellen [5]. Diese Studien belegen, dass NAC die Substratauswahl von anderen Ribosomen-assoziierten Faktoren entscheidend beeinflusst, wofür seine einzigartige Fähigkeit, Substrate bereits im ribosomalen Tunnel und damit als erster Faktor zu binden, notwendig zu sein scheint. Dies wurde anhand einer NAC-Mutante gezeigt, die nicht im Tunnel binden und unspezifische Ribosom-Sec61-Interaktionen nicht mehr inhibieren kann [3]. Die berechtigte Frage, wie NAC reagiert, wenn ein Ribosom ein ERSubstrat synthetisiert, ist nicht abschließend geklärt. Es wird angenommen, dass NAC ER-Signalsequenzen erkennt, durch Konformationsänderung die SRP-Bindungsstelle auf den Ribosomen freigibt und anschließend durch SRP verdrängt wird.
Weitere Studien weisen auch auf eine Funktion von NAC beim mitochondrialen Proteintransport hin. Der Proteinimport in Mitochondrien erfolgt generell auf posttranslationale Weise [6]. Eine Studie an Hefezellen zeigte jedoch, dass der ko-translationale Proteintransport ausgeprägter ist, als bisher angenommen, und insbesondere Proteine der inneren Mitochondrienmembran einschließt [7]. Wie die Ribosomen zur Translokase der äußeren Mitochondrienmembran, dem TOMKomplex, gebracht werden, ist nicht gut verstanden. Ähnlich wie beim ER-Transport spielt die Erkennung von Signalsequenzen in Polypeptiden eine wichtige Rolle. Diese mitochondrialen Targeting-Sequenzen (MTS) sind jedoch im Gegensatz zu den hydrophoben ERSignalsequenzen positiv geladen [6, 7]. Es gibt Hinweise, dass NAC MTS-Substrate erkennt und den Transport der Ribosomen zu den Mitochondrien reguliert (Abb. 2B, rechts). Hefemutanten ohne NAC haben weniger Ribosomen an Mitochondrien gebunden und zeigen mitochondriale Defekte, welche auch in Säugerzellen nachgewiesen wurden [1]. Interessanterweise bindet NAC in der Hefe an das mitochondriale Membranprotein OM14, das als Mitochondrienrezeptor für NAC-gebundene Ribosomen dienen könnte [8]. Darüber hinaus wurde gezeigt, dass sowohl NAC als auch OM14 den mitochondrialen Proteinimport fördern. NAC könnte also als ko-translationaler Transportfaktor fungieren, ähnlich der Rolle von SRP im ERTransport. Im Gegensatz zu SRP bindet NAC jedoch nicht selektiv, sondern global an neu synthetisierte Substrate, einschließlich cytosolischer, ER- und mitochondrialer Proteine [1]. Dies wirft die Frage auf, wie es NAC gelingt, ein MTS-Transportsignal spezifisch zu erkennen und an den TOM-Komplex weiterzuleiten. Eine Möglichkeit wäre, dass NAC eine MTS-spezifische Konformation annimmt, welche von einem mitochondrialen Rezeptor erkannt wird. Ein Zusammenspiel von NAC mit einem weiteren unbekannten Transportfaktor, der MTS-Substrate erkennt, ist ebenfalls denkbar.

\section{Hier steht eine Anzeige.}

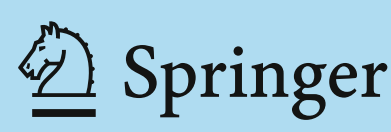




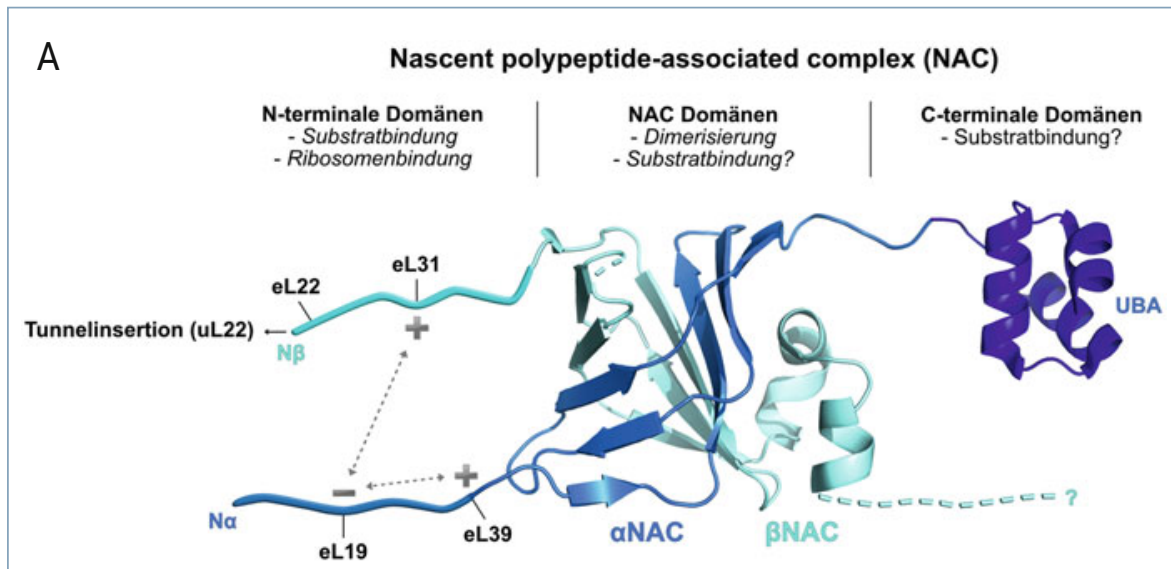

B

Sensing-Konformation

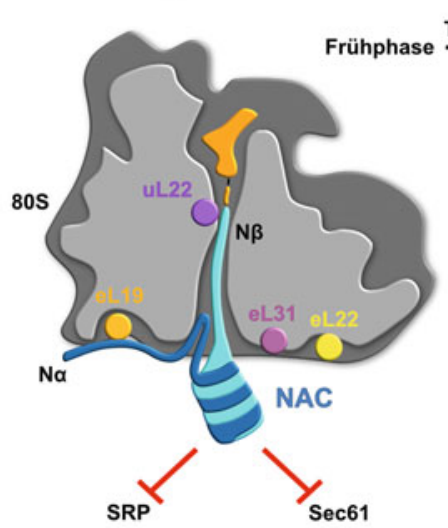

Sorting-Konformation

$\stackrel{\text { Translation }}{\longrightarrow}$ Spätphase

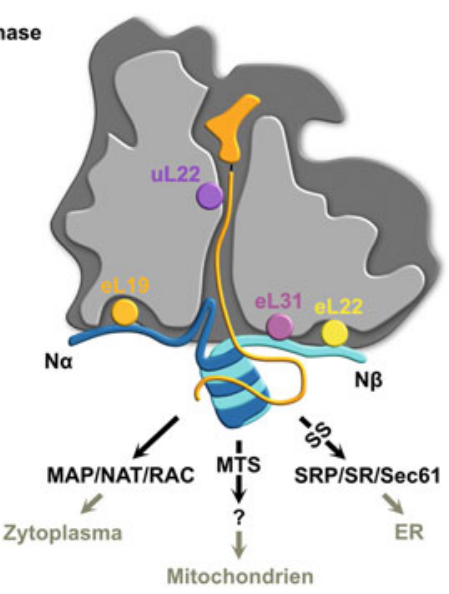

$\triangle$ Abb. 2: Struktureller Aufbau und Ribosomenbindung von NAC. A, Funktioneller Aufbau von NAC. Ribosomenkontakte (eL22, eL31 usw.) sowie geladene Sequenzmotive (+/-) der N-terminalen Domänen ( $N \alpha, N \beta)$ sind angezeigt. B, Ribosomenbindung von NAC in der Frühphase (links) und Spätphase (rechts) der Translation. In der Frühphase, wenn der ribosomale Tunnel nicht von einem Polypeptidsubstrat besetzt ist, führt NAC die flexible N $\beta$-Domäne tief in den Tunnel ein und erkennt Substrate direkt bei der Geburt in der Nähe des Peptidyltransferase-Zentrums. In der sensing-Konformation blockiert NAC die Ribosomenbindung von SRP und Sec61, um einen fehlerhaften Proteintransport zu verhindern. Mit fortschreitender Translation begleitet NAC die wachsende Polypeptidkette zum Tunnelausgang, wo N $\beta$ eine alternative Bindung auf der Ribosomenoberfläche eingeht. In der sorting-Konformation reguliert NAC den Zugang anderer Proteinbiogenesefaktoren, um die korrekte Proteinfaltung im Cytoplasma und den Proteintransport zu den Mitochondrien und dem ER zu gewährleisten. MTS: mitochondriale Targeting-Sequenz; SS: ERSignalsequenz (weitere Abkürzungen siehe Abb. 1).

\section{Proteinfaltung}

Die Synthese und anschließende Faltung von Proteinen ist aufgrund der hohen Molekülkonzentration im Cytoplasma ein äußerst fehleranfälliger Prozess. Nach Verlassen des Ribosomentunnels zeigt die noch lang gestreckte Polypeptidkette hydrophobe Oberflächen, die mit anderen Zellmolekülen unspezifisch interagieren können, was zu Proteinfehlfaltung und -aggregation führen kann. Die Proteinaggregation steht in einem kausalen Zusammenhang mit der Entstehung verschiedener degenerativer Krankheiten beim Menschen, was die Bedeutung von Proteinfaltungsprozessen für das Überleben von Zellen unterstreicht. Daher besitzen alle Zellen ein ausgeklügeltes Netzwerk von molekularen Chaperonen, die spezifisch unstrukturierte Polypeptide binden und deren Faltung fördern, indem sie unspezifische Wechselwirkungen mit anderen Molekülen verhindern. Einige dieser Chaperon- systeme, wie z. B. der Triggerfaktor in Bakterien oder RAC in Eukaryoten, binden dabei direkt an den ribosomalen Tunnelausgang, um neu synthetisierte Proteine ko-translational bei der Faltung zu unterstützen [1].

Aufgrund seiner Bindung direkt am Ausgang des ribosomalen Tunnels und seiner Fähigkeit, mit naszierenden Polypeptidketten $\mathrm{zu}$ interagieren, wurde NAC von jeher eine Funktion als Chaperon zugeschrieben. Eindeutige Beweise für diese Rolle wurden jedoch erst kürzlich erbracht. In einer in vitro-Studie wurde erstmals direkt nachgewiesen, dass NAC verschiedene, strukturell unterschiedliche Chaperon-Substrate bindet und vor Aggregation schützt, wie z. B. Polyglutamin-Huntingtin, $\alpha$-Synuklein und $A \beta 40$, die zelltoxische Proteinaggregate bilden können und mit verschiedenen neurodegenerativen Erkrankungen wie Alzheimer und Parkinson assoziiert sind [9]. Diese ChaperonAktivität von NAC wurde in vivo im Fadenwurm C. elegans sowie in Maus-Nervenzellen bestätigt (Abb. 3A), überraschenderweise ist sie aber bei Polyglutamin-Proteinen in vivo unabhängig von der Ribosomenbindung [9]. Man fand, dass ein großer Teil der NAC-Moleküle in der Zelle nicht an Ribosomen gebunden ist, posttranslational an unstrukturierte Proteine bindet und diese so vor Aggregation schützt. Interessanterweise wurde eine wichtige Interaktionsstelle für Polyglutamin-Proteine in der positiv geladenen Ribosomenbindungsdomäne der $\beta$-Untereinheit von NAC identifiziert, welche auch naszierende Substrate im Ribosomentunnel bindet (Abb. 3B). Dies legt nahe, dass NAC über diese Domäne auch ko-translational Substrate bindet, um neu synthetisierte Proteine bei der Faltung zu unterstützen. Diese Hypothese wird durch Studien gestützt, die zeigen, dass NAC Polypeptidketten am Ribosom stabilisiert und vor vorzeitigem Abbau schützt [1]. NAC bindet naszierende Polypeptide über die $\alpha$ - und $\beta$-Untereinheit, was auf eine multimodale Substratbindung schließen lässt. Zusammengefasst weisen diese Befunde auf einen Chaperon-Mechanismus ähnlich dem des Triggerfaktors in Bakterien hin, der über mehrere Bindungsstellen naszierende Polypeptide am Tunnelausgang bindet und so ihre potenzielle Fehlfaltung verhindert.

\section{Danksagung}

Die Autoren danken der DFG für die Unterstützung im Rahmen des SFB969 und C. Schlatterer und E. Oberer-Bley für ihre Hilfe bei der Erstellung des Manuskripts. 
A

\section{Polyglutamin-Aggregation}
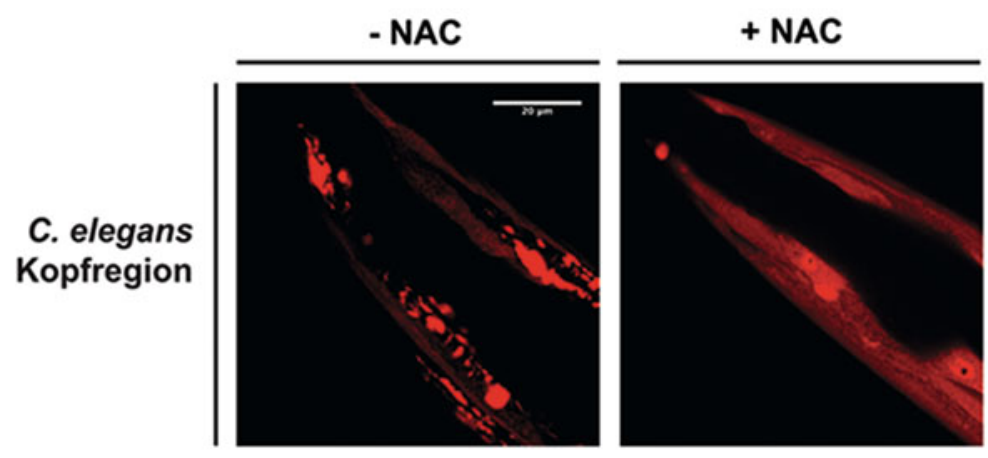

B

\section{S}
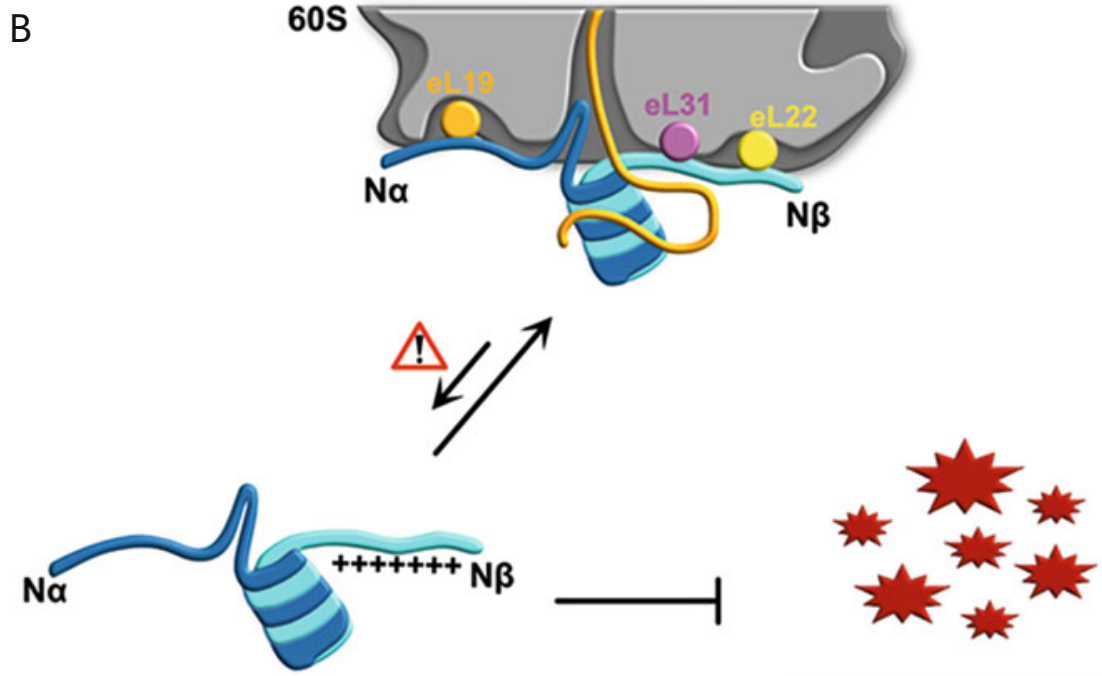

Polyglutamin-Protein Aggregate

$\triangle$ Abb. 3: Molekulare Chaperon-Aktivität von NAC. A, Fluoreszenzmikroskopische Aufnahmen von Polyglutamin-Proteinen (rot) in der Kopfregion von Caenorhabditis elegans. Toxische Proteinaggregate (links) verschwinden nach der Überexpression von NAC (rechts). B, Schematische Darstellung der posttranslationalen Chaperon-Aktivität von NAC. Unter Stressbedingungen verhindert NAC über die positiv geladene Ribosomenbindedomäne (N $\beta$ ) die Bildung von toxischen Polyglutamin-Aggregaten.

\section{Literatur}

[1] Deuerling E, Gamerdinger M, Kreft SG (2019) Chaperone interactions at the ribosome. Cold Spring Harb Perspect Biol 11, doi: 10.1101/cshperspect.a033977

[2] Wiedmann B, Sakai H, Davis TA et al. (1994) A protein complex required for signal-sequence-specific sorting and translocation. Nature 370:434-440

[3] Gamerdinger M, Kobayashi K, Wallisch A et al. (2019) Early scanning of nascent polypeptides inside the ribosomal tunnel by NAC. Mol Cell 75:996-1006
[4] Walter P (1994) Signal sequence recognition and protein targeting to the endoplasmic reticulum membrane. Annu Rev Cell Biol 10:87-119

[5] Gamerdinger M, Hanebuth MA, Frickey T et al. (2005) The principle of antagonism ensures protein targeting specificity at the endoplasmic reticulum. Science 348:201-207

[6] Neupert W, Herrmann JM (2007) Translocation of proteins into mitochondria. Annu Rev Biochem 76:723-749
[7] Williams CC, Jan CH, Weissman JS (2014) Targeting and plasticity of mitochondrial proteins revealed by proximityspecific ribosome profiling. Science 346:748-751

[8] Lesnik C, Cohen Y, Atir-Lande A et al. (2014) OM14 is a mitochondrial receptor for cytosolic ribosomes that supports co-translational import into mitochondria. Nat Commun 5: 571

[9] Shen K, Gamerdinger M, Chan R et al. (2019) Dual role of ribosome-binding domain of NAC as a potent suppressor of protein aggregation and aging-related proteinopathies. Mol Cell 74:729-741

Funding: Open Access funding provided by Projekt DEAL.

Open Access: Dieser Artikel wird unter der Creative Commons Namensnennun 4.0 International Lizenz veröffentlicht, welche die Nutzung, Vervielfältigung, Bearbeitung, Verbreitung und Wiedergabe in jeglichem Medium und Format mäß nennen einen maßs nennen, einen Link zur Creative Commons Lizenz beilugen und angeben, o sonstiges Drittmaterial unterliegen. Dienfalls der arenanten Creative Comm Lizenz, sofern sich aus der Abbildungslegende nichts anderes ergibt. Sofern das betreffende Material nicht unter der genannten Creative Commons Lizenz steht und die betreffende Handlung nicht nach gesetzlichen Vorschriften erlaubt ist, ist für die oben aufgeführten Weiterverwendungen des Materials die Einwilligung des jeweiligen Rechteinhabers einzuholen. Weitere Details zur Lizenz entnehme Sie bitte der Lizenzinformation auf http://creativecommons.org/licenses/ by $/ 4.0 /$ deed.de.

Korrespondenzadresse:

Dr. Martin Gamerdinger

FB Biologie/Molekulare Mikrobiologie Universität Konstanz

Fach 606

D-78457 Konstanz

martin.gamerdinger@uni-konstanz.de

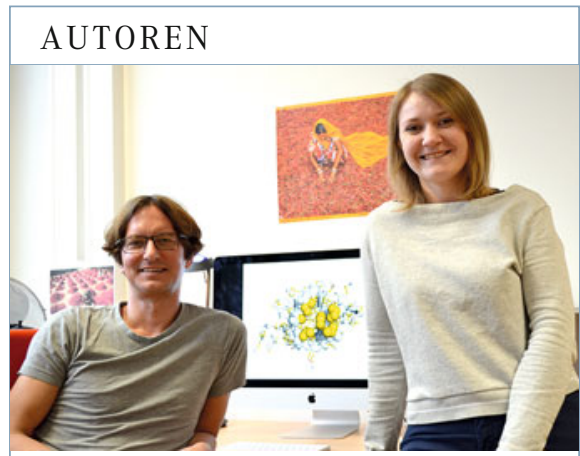

Martin Gamerdinger und Karina Gense.

Martin Gamerdinger

1999-2009 Biologiestudium und Promotion am Institut für Pathobiochemie an der Universität Mainz unter Anleitung von Prof. Dr. C. Behl. 2009-2011 Postdoktorand an der Universität Mainz. 2012-2015 Postdoktorand an der Universität Konstanz im Labor von Prof. Dr. E. Deuerling. Seit 2016 unabhängiger Nachwuchsgruppenleiter an der Universität Konstanz.

\section{Karina Gense}

2010-2016 Life-Science-Studium an der Universität Konstanz. Seit 2016 Promotion am Lehrstuhl für Molekulare Mikrobiologie unter Anleitung von Prof. Dr. E. Deuerling, Universität Konstanz. 